www.jmscr.igmpublication.org

Impact Factor 5.84

Index Copernicus Value: 71.58

ISSN (e)-2347-176x ISSN (p) 2455-0450

crossref DOI:_https://dx.doi.org/10.18535/jmscr/v6i1.80

\author{
Journal Of Medical Science And Clinical Research \\ IGM Publication \\ An Official Publication of IGM Publication
}

\title{
Role of Conventional IVU (Intra Venous Urography) and Computed Tomography in Patients of Urinary Tract Calculopathy
}

\begin{abstract}
Authors
Varun Goyal $^{1}$, Pramod Shaha ${ }^{2}$, Kulamani Sahoo ${ }^{3}$, Dhruv Aggarwal ${ }^{4}$, Natasha Vijayendran ${ }^{5}$, Harshwardhan Thite ${ }^{6}$, Varun Tyagi ${ }^{7}$

1,4,6,7 Junior Resident, Radiodiagnosis, Krishna Institute of Medical Sciences, Karad, Maharashtra

${ }^{2}$ Professor, Radiodiagnosis, Krishna Institute of Medical Sciences, Karad, Maharashtra

${ }^{3}$ Professor and Head of the Dept, Radiodiagnosis, Krishna Institute of Medical Sciences, Karad, Maharashtra

${ }^{5}$ Junior Resident, Dermatology, Krishna Institute of Medical Sciences, Karad

Corresponding Author

Varun Goyal

Department of Radiodiagnosis, Krishna Institute of Medical Sciences, Karad, Maharashtra, India-415110

Email: varun.or.goyal@ gmail.com, Phone No- + 917057871508

Abstract

Background: From calculus disease to hematuria, imaging has been of great importance in the diagnosis of many diseases of the urinary tract. Advances in imaging technology have changed the practice of uroradiology significantly. CT urography represents one of the most advanced developments in imaging the urinary tract to date. Excretory urography has been the initial modality for upper tract imaging in patients with hematuria, flank pain, and other urologic diseases for the past 5 decades. With the recent introduction of multi-detector row helical CT, the uroradiologic evaluation of patients with common and complex disease is changing rapidly. This study is an attempt to review the role of IVU or CT Urography, should supercede as investigation of choice in varied settings and evaluate the obstructed tract anatomy in patients with non-functioning kidneys and various urinary tract disorders.

Materials And Method: It was a hospital based, comparative study, with a sample size of 60 patients, during the period of 2 years, in the department of Radio-Diagnosis, Krishna institute of Medical sciences, karad.

Patients referred with acute flank pain, hematuria or ultrasonologically detected cases of urinary tract calculopathy were included in the study.

Patients were randomly allocated in following two groups of 30 patients - IVU Group and CT Urography Group.

Then according to the analysis of both the groups, the results were compiled and compared statistically and graphically to depict the individualistic role of each modality, their cons and pros in varied settings and management in patients with urinary tract calculopathy.

Results: Amongst the two groups the number of patients whose urinary stones were detected was higher in the CT Urography group than IVU group. Urography group had a higher proportion of Renalstones, mid \& lower ureteral stones, than the IVU group. The mean size of obstructing calculi detected by IVU was $6.03 \pm 1.08 \mathrm{~mm}$ as compared $6.80 \pm 2.01 \mathrm{~mm}$ as detected by CT Urography. The accuracy of detecting etiology by CT Urography was $100 \%$ as compared to $66.7 \%$ of IVU.CT was able to differentiate the acute and chronic changes in the renal parenchyma due to obstructive uropathy in many cases however IVU was unable to do so.

Conclusion: CTU is better than IVU in detection of urinary stone, saves time and is cost effective however IVU involves less radiation dose. From this study we conclude that CT provides a better diagnostic information in the patients with urinary tract calculopathy.

Keywords: IVU, Computed Tomography, Caluculopathy, IVP, CT KUB.
\end{abstract}




\section{Introduction}

Imaging of the upper urinary tract has traditionally been the purview of intravenous (IV) urography, but over the last decade, computed tomography urography (CTU) has become the modality of choice in imaging the urinary tract.

From calculus disease to hematuria, imaging has been of great importance in the diagnosis of many diseases of the urinary tract. Advances in imaging technology have changed the practice of uroradiology significantly.

CT urography represents one of the most advanced developments in imaging the urinary tract to date.

Excretory urography has been the initial modality for upper tract imaging in patients with hematuria, flank pain, and other urologic diseases for the past 5 decades. With the recent introduction of multidetector row helical CT, the uroradiologic evaluation of patients with common and complex disease is changing rapidly

Hence the present study was done to review the role of IVU or CT Urography, should supercede as investigation of choice in varied settings and evaluate the obstructed tract anatomy in patients with non-functioning kidneys and various urinary tract disorders.

\section{Materials and Methods \\ Patients}

- A hospital based study was undertaken on 60 patients to assess the role of Conventional IVU (Intra-venous urography) and Computerized Tomography in patients of urinary tract calculopathy. Both sexes, Patients within range Serum creatinine $(0.8-1.4 \mathrm{mg} / \mathrm{dl})$ and all age groups were included in the study.

- Patients having Pelvicmass, not willing to participate in the study and not having there serum creatinine in normal range were excluded out of this study.

\section{Methodology}

Patients referred with acute flank pain, hematuria or ultrasonologically detected cases of urinary tract calculopathy were included in the study.

Patients were randomly allocated in following two groups of 30 patients - IVU Group and CT Urography Group and their Study based on their modality was done and later on the results were compared.

\section{Equipment Used}

- Multi detector 16 slice Siemens Emotion CT

- Conventional IVU (Siemens Polydros, GE TEJAS XR 6000)

\section{IVU - Intra venous Urography}

- Patient was asked to be Nil per-oral (NPO) overnight and the study was performed the coming morning.

- Patient was asked to lie down in the supine position on the table with pelvis at the cathode side of the tube.

- Plain abdominal film (ScoutImage) was taken from the level of xiphisternum to anterior superior iliac spine.

- Contrast media is injected intravenously ( $1 \mathrm{ml} / \mathrm{Kg}$ ) into a prominent vein in the arm.

- Series of films were taken at 1 minute (Nephrogram), 5 minutes, 15 minutes, 30 minutes and Post-Void films.

- Further delayed images were also taken if required.

\section{Computed Tomography Urography (CTU)}

- CTU was performed with a 16 slice Siemens Emotion CT from the level of the kidneys to the pubic symphysis in breathhold status, with the following parameters: beam collimation $5 \mathrm{~mm} \times 1.25 \mathrm{~mm}$; pitch 6 ; scan time about $20 \mathrm{~s}$; and post contrast images acquired. Subsequent curved threedimensional multiplanar reconstruction (MPR) focusing on the ureter of the symptomatic side was performed on a compatible workstation by an experienced 
CT technician. By manually selecting a point within the center of the ureteric lumen on sequential axial images, the renal collecting system could be demonstrated completely from the level of the renal pelvis to the urinary bladder.

\section{Results}

- Majority of the patients $(36.7 \%)$ in IVU Group were from the age group of 41-50 years followed by $26.7 \%$ patients from the age group of 51-60 years, $20 \%$ patients from the age group of $61-70$ years, $10 \%$ patients from the age group of 31-40 years and $6.6 \%$ patients from the age group of 21-30 years. The mean age of patients in Group A was $48.8 \pm 11.82$ years.

- Majority of the patients (33.3\%) in Computerized Tomography Urography Group were from the age group of 41-50 years by $30 \%$ patients from the age group of 51-60 years, $23.4 \%$ patients from the age group of $61-70$ years, $10 \%$ patients from the age group of 31-40 years and $3.3 \%$ patients from the age group of 21-30 years. The mean age of patients in Group A was $51.3 \pm 11.23$ years. There was no significant difference between the groups.

- Majority of the patients in both groups were male. There were $66.7 \%$ and $70 \%$ male in IVU Group and CT Urography Group respectively whereas female constituted $33.3 \%$ and $30 \%$ of the study group respectively. There was no significant difference in both the groups.

- Most of the patients had more than one symptom. The most common symptom in both groups was flank pain $(90 \%$ and $86.7 \%)$ followed by abdominal pain $(66.7 \%$ and $70 \%)$, hematuria $(30 \%$ and $26.7 \%)$, micturition disturbances $(23.3 \%$ and $20 \%$ ), lump (16.7\% and $13.3 \%)$ and fever $(6.6 \%$ and $10 \%)$. There was no significant difference in both the groups.
- There was equal distribution of disease in both the groups. There was no significant difference in both the groups.

- The distribution of various etiologies in IVU group are characterized as follows: Obstructive 33.3\%, Neoplastic 10\%, Infective $6.6 \%$, Post-operative/Postintervention $3.3 \%$, Congenital $3.3 \%$, Urinary Bladder Pathologies 3.3\%, Renal Cystic Disease 3.3\%, Extra urinary 3.3\% and in CT Urography group they are characterized as Obstructive $46.7 \%$, Neoplastic $13.3 \%$, Infective $10 \%$, Postoperative/Post-intervention $10 \%$, Congenital $6.6 \%$, Urinary Bladder Pathologies 6.6\%, Renal Cystic Disease $3.3 \%$ and Extra urinary $3.3 \%$ respectively. The most common cause was obstructive calculi followed by neoplastic calculi.

- A total of 40 urinary stones were detected in 30 patients. The number of patients whose urinary stones were detected was higher in the CT Urography group than IVU group $(75 \%$ versus $60 \%, \mathrm{p}<0.05)$. The CT Urography group had a higher proportion of kidney stones and mid ureteral stones, than the IVU group (20\% vs. $12.5 \% ; 16.7 \%$ vs. $8.3 \%, \mathrm{p}<0.05$ respectively); however, the proportion of distal ureteral stones was lower in CT Urography group than in IVU group.

- There were 4 patients with multiple stones. CT Urography detected multiple stones in 3 patients whereas IVU detected multiple stones in 2 patients. This difference was statistically not significant.

- 11 (33.7\%) and 12 (40\%) patients respectively in IVU and CT Urography group had obstructing calculi more than 8 $\mathrm{mm}$, making passage through the urinary tract difficult. The mean size of obstructing calculi detected by IVU was $6.03 \pm 1.08 \mathrm{~mm}$ as compared $6.80 \pm 2.01 \mathrm{~mm}$ as detected by CT Urography. This difference was statistically significant. 
- $3(10 \%)$ and $6(20 \%)$ patients respectively in IVU and CT Urography group underwent surgical management whereas $27(90 \%)$ and 24 (80\%) patients underwent non-surgical management. This difference was statistically not significant.

- The accuracy of detecting etiology by CT Urography was $100 \%$ as compared to
$66.7 \%$ of IVU. There was significant difference in the accuracy of CT Urography as compared to IVU.

Table 1 Distribution of patients according to Location of Stone

\begin{tabular}{|l|c|c|c|c|c|c|}
\hline \multirow{2}{*}{ Location of Stone } & \multicolumn{2}{|c|}{ IVU } & \multicolumn{2}{c|}{ CT Urography } & \multirow{2}{*}{ Total } & \multirow{2}{*}{ p Value } \\
\cline { 2 - 5 } & $\mathbf{N}$ & $\mathbf{\%}$ & $\mathbf{N}$ & $\mathbf{\%}$ & & \\
\hline Kidney & 3 & $12.5 \%$ & 6 & $20 \%$ & 8 & $\mathbf{p}<\mathbf{0 . 0 5}$ \\
\hline Proximal Ureter & 8 & $33.3 \%$ & 10 & $33.3 \%$ & 12 & $\mathrm{p}>0.05$ \\
\hline Middle Ureter & 2 & $\mathbf{8 . 3 \%}$ & 5 & $16.7 \%$ & 7 & $\mathbf{p}<\mathbf{0 . 0 5}$ \\
\hline Distal Ureter & 09 & $40.9 \%$ & 11 & $34.3 \%$ & 13 & $\mathbf{p}<\mathbf{0 . 0 5}$ \\
\hline Total & 22 & $100 \%$ & 32 & $100 \%$ & 40 & \\
\hline
\end{tabular}

Table 2: Distribution of patients according to Size of Obstructing Calculi

\begin{tabular}{|l|c|c|c|c|c|}
\hline \multirow{2}{*}{ Size $(\mathbf{m m})$} & \multicolumn{2}{|c|}{ IVU } & \multicolumn{2}{c|}{ CT Urography } & \multirow{2}{*}{ p Value } \\
\cline { 2 - 5 } & $\mathbf{N}$ & $\mathbf{\%}$ & $\mathbf{N}$ & $\mathbf{\%}$ & \\
\hline $\mathbf{0 - 4}$ & 10 & $33.3 \%$ & 9 & $30 \%$ & \multirow{2}{*}{$\mathbf{p}<\mathbf{0 . 0 5}$} \\
\hline $\mathbf{4 - 8}$ & 9 & $30 \%$ & 9 & $30 \%$ & \\
\hline$>\mathbf{8}$ & 11 & $36.7 \%$ & 12 & $40 \%$ & \\
\hline Total & 30 & $100 \%$ & 30 & $100 \%$ & \\
\hline Mean \pm SD & \multicolumn{2}{|c|}{$6.03 \pm 1.08$} & \multicolumn{2}{|c|}{$6.80 \pm 2.01$} & \multirow{2}{*}{$\mathbf{p}<\mathbf{0 . 0 5}$} \\
\hline
\end{tabular}

Figure-1

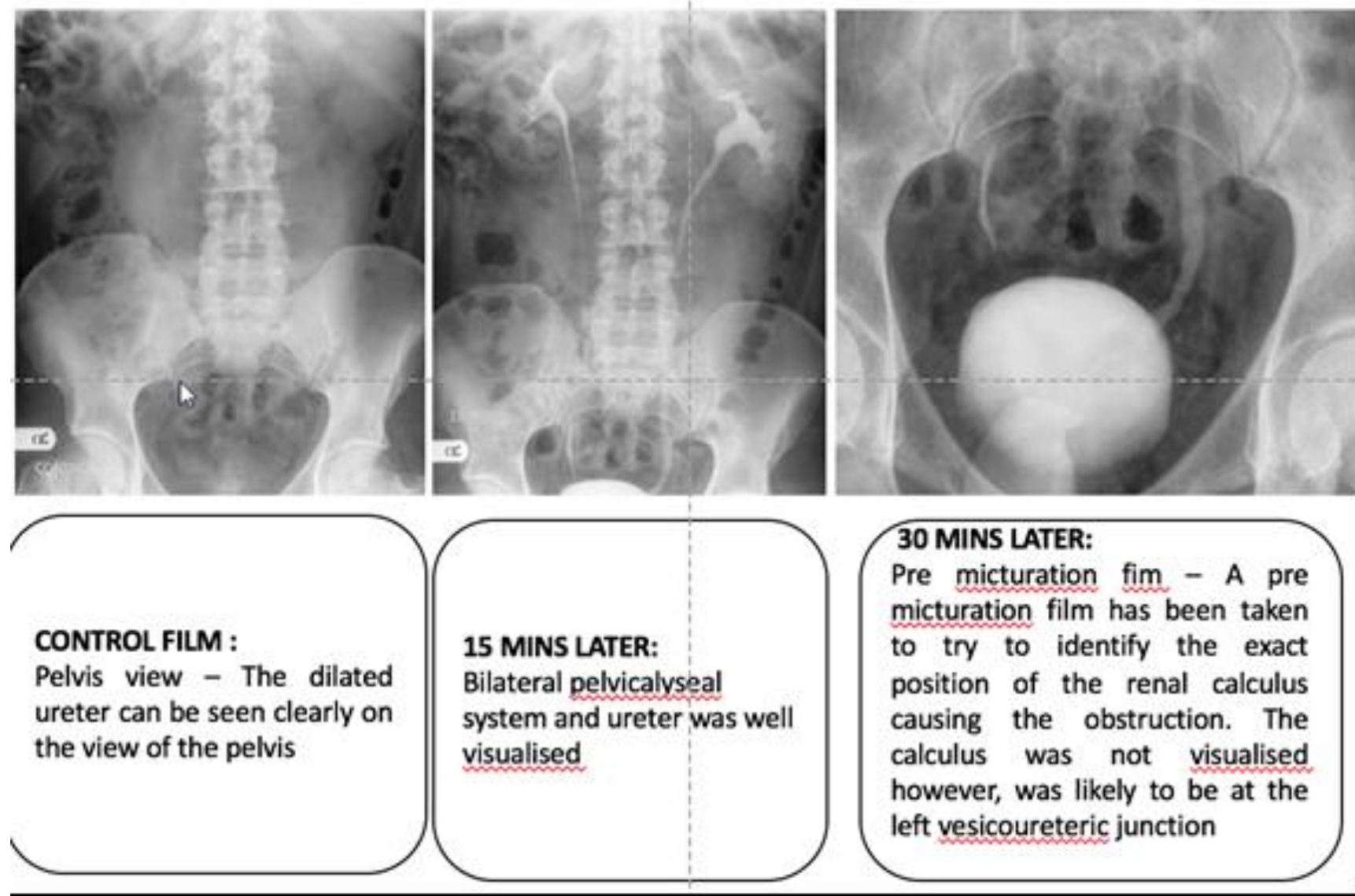




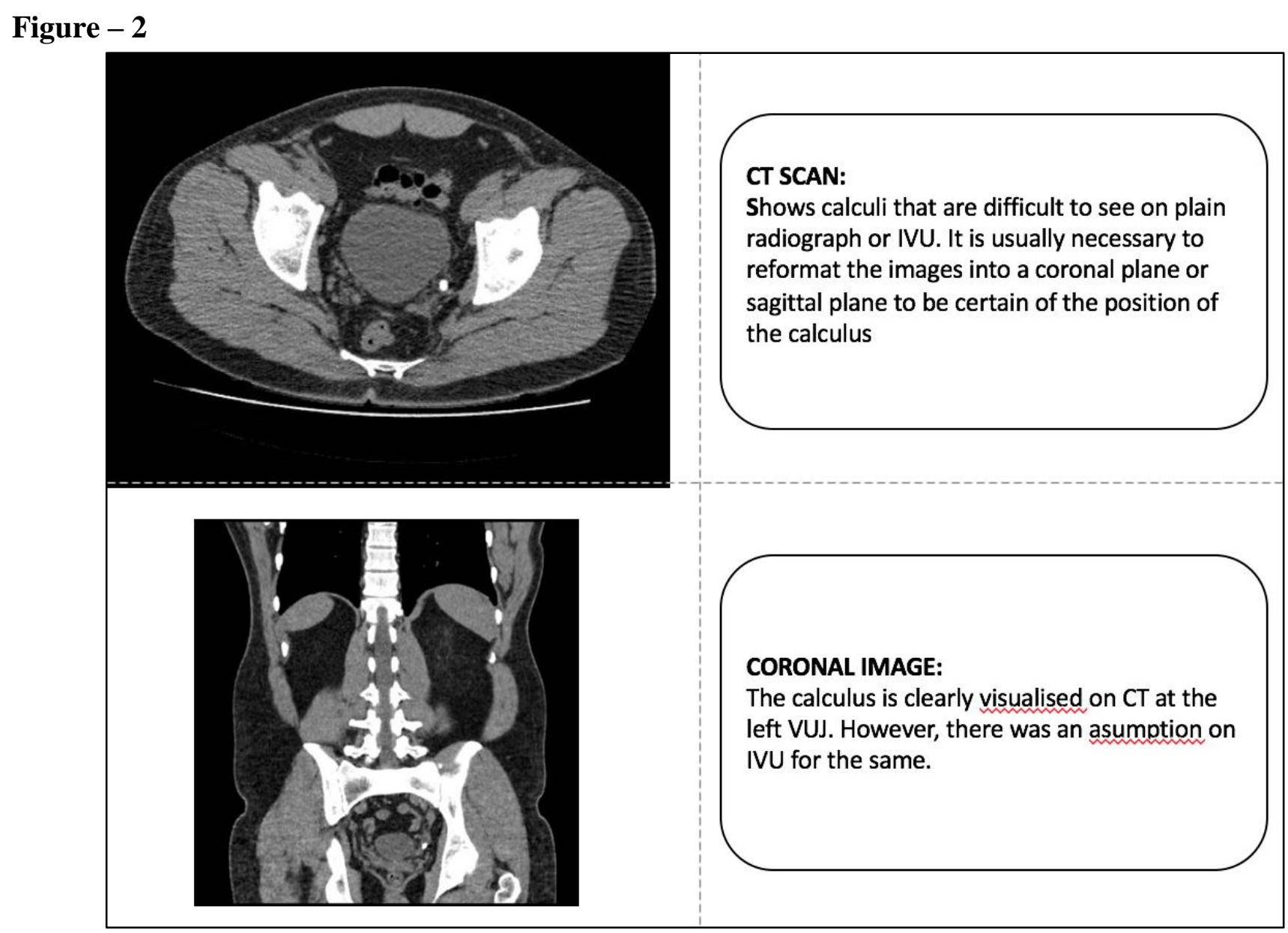

Figure - 3

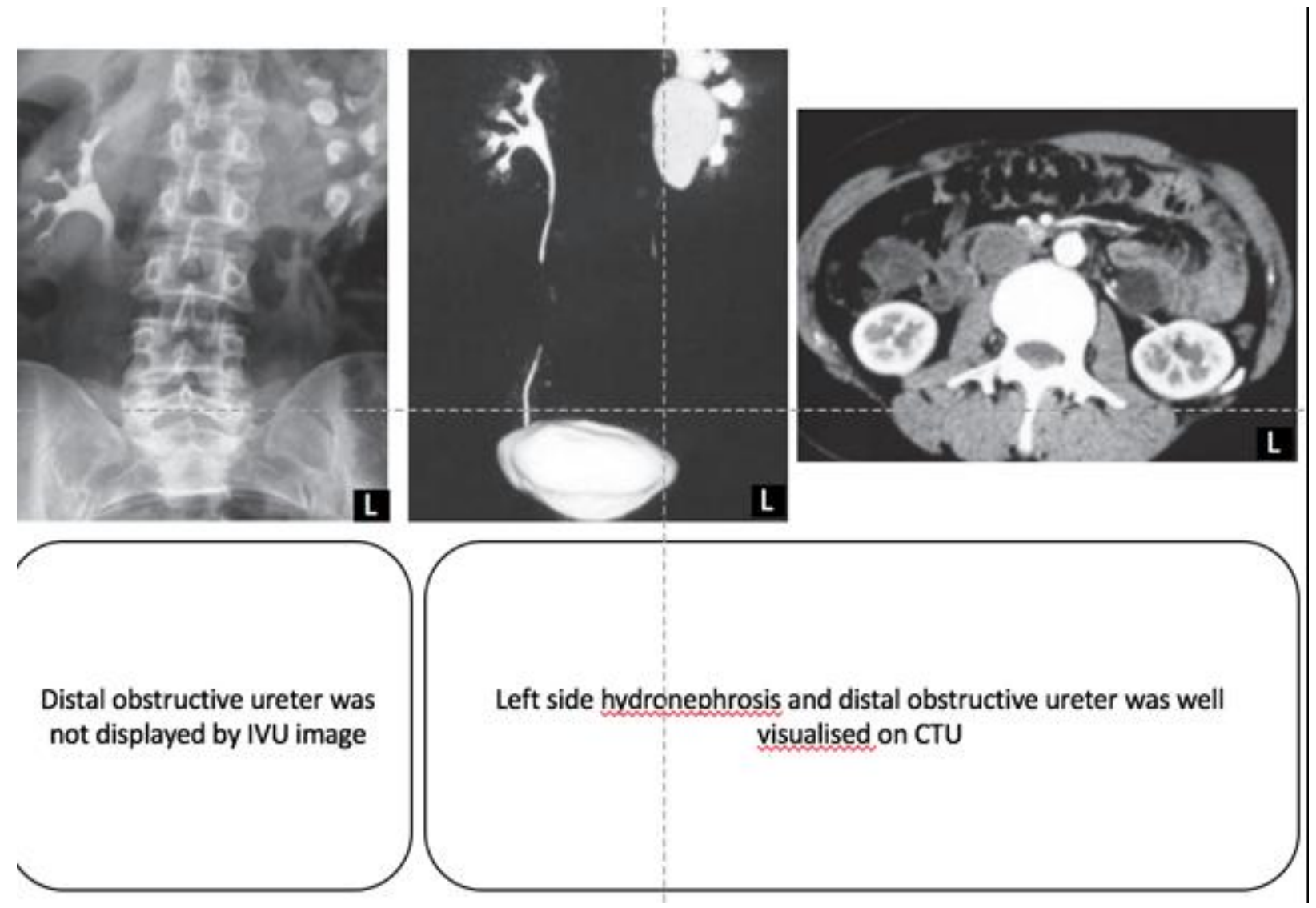




\section{JMSCR Vol||06||Issue||01||Page 31885-31894||January}

Figure 4
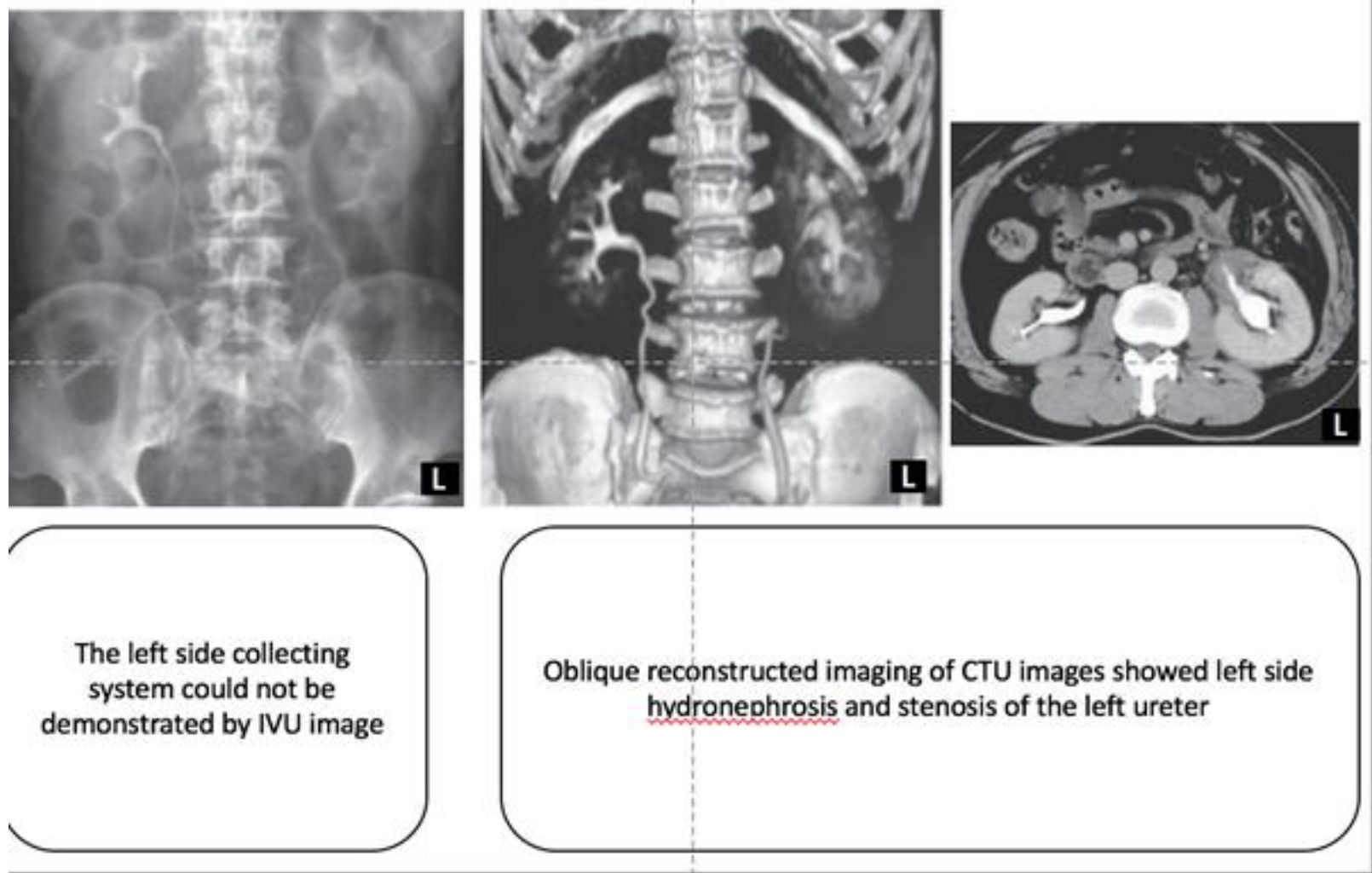

Figure 5

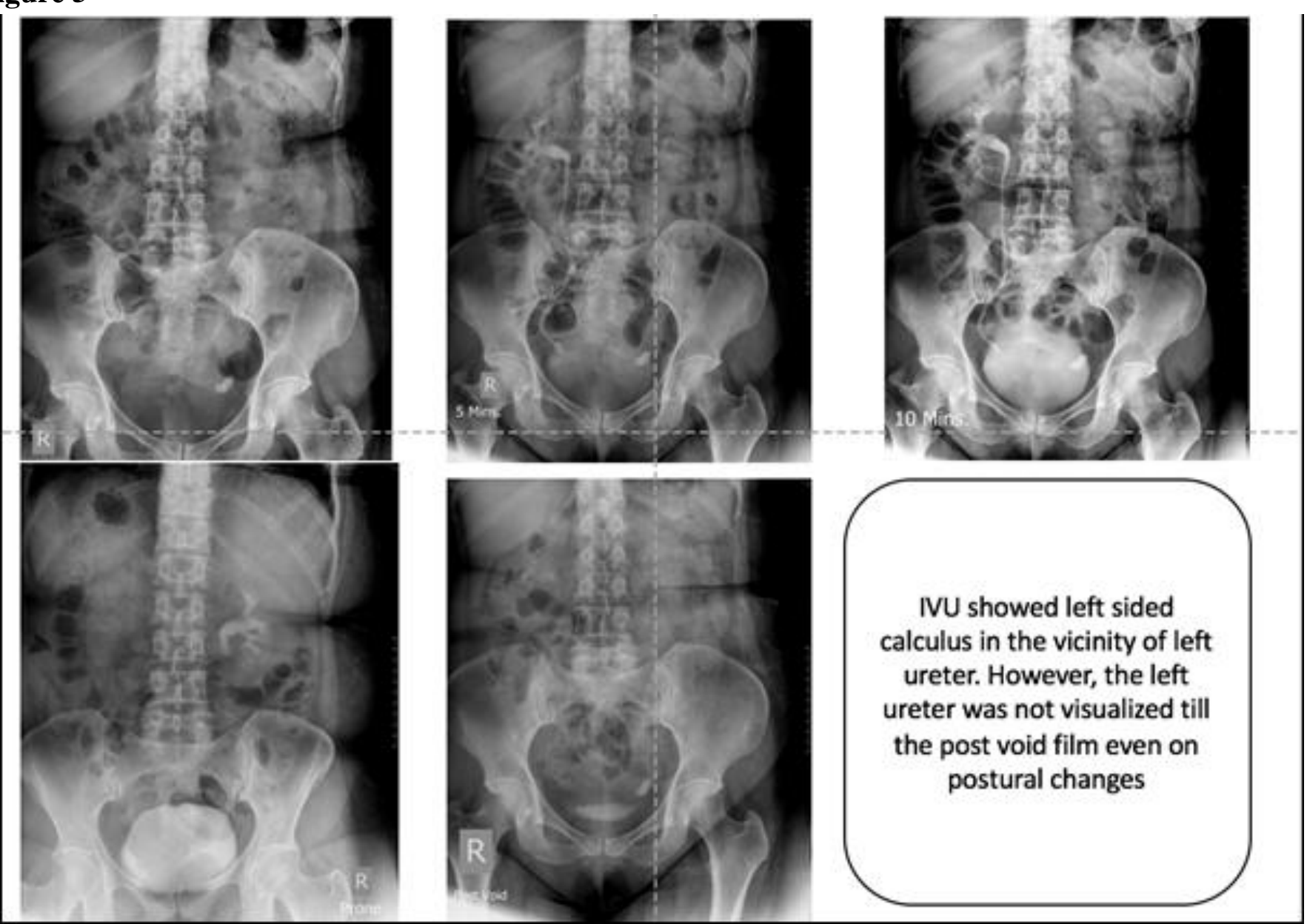



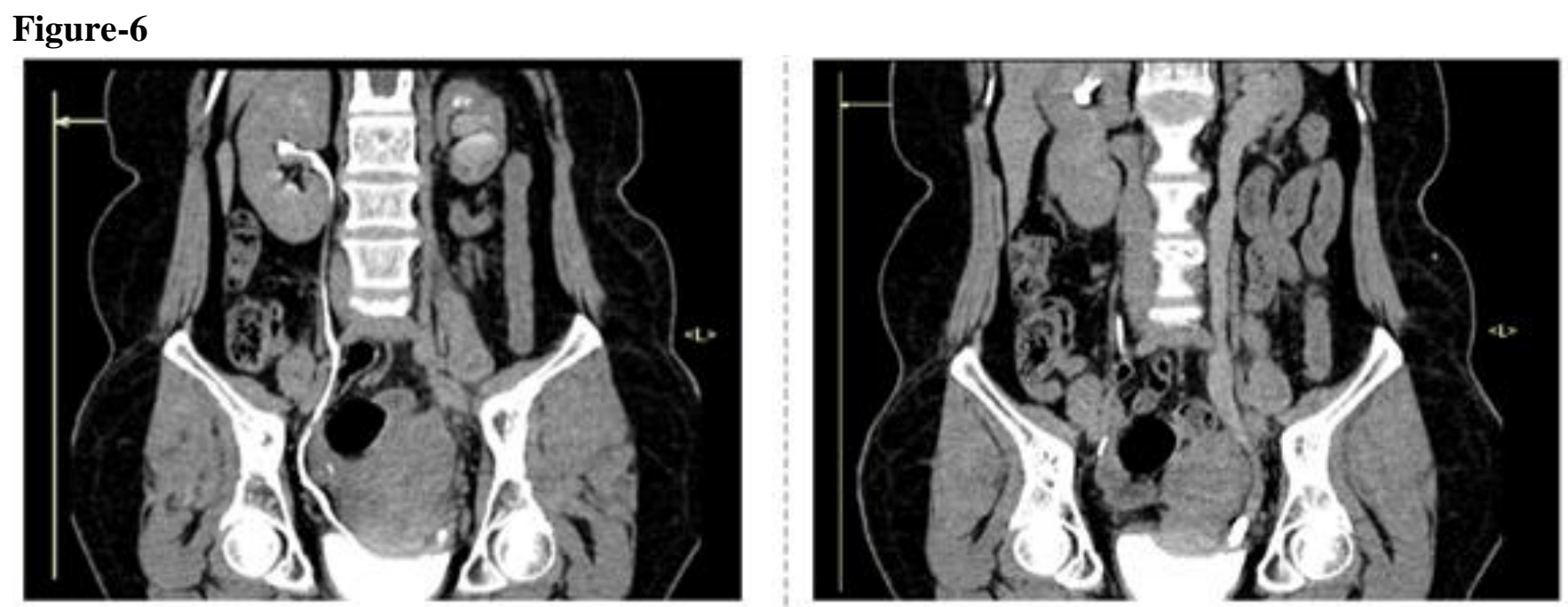

MPR CTU Showed left sided hydroureter with a calculus at thee distal ureter

\section{Discussion}

A prospective comparative study was conducted with 60 patients to review the role of IVU or CT Urography and evaluate the obstructed tract anatomy in patients with urinary tract calculopathy. Patients were randomly allocated in following two groups of 30 patients - IVU Group and CT Urography Group. Imaging of the urinary tract is pivotal in the diagnosis, management, and follow-up of patients with urolithiasis. A significant drawback of IVU is its failure to differentiate between acute obstruction and residual changes due to previous obstruction. CT has the advantage that it can be used to determine the renal parenchymal attenuation to differentiate between acute and chronic obstruction.

\section{Age \& Sex}

In the present study, mean age of patients was 50.03 years and majority of the patients in both groups were male. There were $66.7 \%$ and $70 \%$ male in IVU Group and CT Urography Group respectively whereas female constituted $33.3 \%$ and $30 \%$ of the study group respectively. Lim GS et alretrospective study investigating the changing pattern in the use of intravenous pyelogram (IVP), conventional computed tomography (CT), and non-contrast-enhanced computed tomography (NECT) for evaluation of patients with acute flank pain reported mean age of the patients was 45.10 years and 1,458 patients (66.9\%) were men. Gupta $\mathrm{R}$ et al in a retrospective study on evaluating the role of multi-detector CT urography for detection of urinary tract abnormalities reported 60 patients, $41(68.33 \%)$ were men and $19(31.66 \%)$ women. The findings in our study was similar to those previously undertaken.

\section{Symptoms}

It was observed in our study that most of the patients had more than one symptom. The most common symptom in both groups was flank pain (90\% and $86.7 \%$ ) followed by abdominal pain (66.7\% and $70 \%$ ), hematuria (30\% and $26.7 \%$ ), micturition disturbances (23.3\% and $20 \%$ ), lump (16.7\% and $13.3 \%)$ and fever (6.6\% and $10 \%)$. Lim GS et al68 in a retrospective study investigating the changing pattern in the use of intravenous pyelogram (IVP), conventional computed tomography (CT), and non-contrastenhanced computed tomography (NECT) found that majority of the patients had acute flank pain as the presenting symptom. Lee DH et al found of the 2,218 patients, no cause of pain was identified in 655 patients $(29.5 \%)$, urolithiasis was identified as the cause of pain in 1,413 patients $(63.7 \%)$, and a non-urolithiasis cause was found in 150 patients (6.8\%). Among the 150 patients $(6.8 \%)$ with a non-urolithiasis cause, 39 causes $(1.8 \%)$ were classified as acutely important, 75 causes $(3.4 \%)$ were classified as follow up recommended, and 36 causes $(1.6 \%)$ were classified as an unimportant cause. No cause of pain was found in 632 patients (28.5\%), urolithiasis was identified as the cause of 
pain in 1,433 patients $(64.6 \%)$, and a nonurolithiasis cause was detected in 153 patients $(6.8 \%)$. The findings in our study was similar to the above studies.

\section{Etiology}

In our study, the distribution of various etiologies in IVU group are characterized as follows: Obstructive 33.3\%, Neoplastic 10\%, Infective 6.6\%, Post-operative/Post-intervention $3.3 \%$, Congenital $3.3 \%$, Urinary Bladder Pathologies 3.3\%, Renal Cystic Disease 3.3\%, Extra urinary $3.3 \%$ and in CT Urography group they are characterized as Obstructive $46.7 \%$, Neoplastic $13.3 \%$, Infective $10 \%$, Post-operative/Postintervention $10 \%$, Congenital $6.6 \%$, Urinary Bladder Pathologies 6.6\%, Renal Cystic Disease $3.3 \%$ and Extra urinary $3.3 \%$ respectively. The most common cause was obstructive calculi followed by others. Gupta $\mathrm{R}$ et alreported 42 cases (70\%) demonstrated urolithiasis abnormality and 18 cases $(30 \%)$ demonstrated non urolithiasis abnormality. Out of 18 cases, $6(10 \%)$ cases demonstrated masses. $8 \quad(13.33 \%)$ cases demonstrated inflammatory changes. Congenital anomalies were found in 4 patients $(6.66 \%)$. This finding correlates with the study by Gupta $\mathrm{R}$ et al.

\section{Laterality}

In our study, in the IVU and CTU group, there were 12 patients with left and right calculi respectively. 3 patients had bilateral calculi. Xie $\mathrm{C}$ et al in a study found in a total of 61 patients, thirty-two had a right-sided UPJO and 29 patients had a left-sided UPJO (Uretero-pelvic Junction obstruction ). This correlates with the study done by $\mathrm{Xie} \mathrm{C}$ et al in which laterality played no significant role.

\section{Multiple Calculi}

In our study, there were 4 patients with multiple stones. CT Urography detected multiple stones in 3 patients whereas IVU detected multiple stones in 2 patients. Khan $\mathrm{N}$ et al observed more number of ureteric stones on CT than IVU at all locations Thus the findings in our study was similar and comparable to the above study.

\section{Site}

In our study, a total of 40 urinary stones were detected in 30 patients. The number of patients whose urinary stones were detected was higher in the CT Urography group than IVU group (75\% versus $60 \%, \mathrm{p}<0.05)$. Lee $\mathrm{DH}$ et al in a retrospective analysis of IVU and NECT found that the number of patients whose urinary stones were detected on an imaging study was higher in the NECT group than in the IVU group (74\% versus $59 \%, p<0.001)$. Thus the findings in our study was similar and comparable to the above study.

\section{Size}

It was observed in the present study that 11 (33.7\%) and 12 (40\%) patients respectively in IVU and CT Urography group had obstructing calculi more than $8 \mathrm{~mm}$, making passage through the urinary tract difficult. The mean size of obstructing calculi detected by IVU was $6.03 \pm 1.08 \mathrm{~mm}$ as compared $6.80 \pm 2.01 \mathrm{~mm}$ as detected by CT Urography. Khan $\mathrm{N}$ et al found mean stone size was $5.3 \mathrm{~mm}$ for stones identified on CT, $6.4 \mathrm{~mm}$ for those identified on IVU and $5.9 \mathrm{~mm}$ for those missed on IVU. Lee DH et al, in a retrospective analysis of IVU and NECT performed in adult patients with suspected renal colic found NECT use resulted in a higher detection rate of renal stones and radiolucent stones than IVU use, and the size of the stones that were detected with NECT was smaller than those that were detected with IVU. The mean urinary stone size was smaller $(3.62 \pm 3.23 \mathrm{~mm}$ versus $4.15 \pm 2.36 \mathrm{~mm}, p<0.001)$ and the stones were more radiolucent $(25.7 \%$ versus $13.2 \%, p<$ 0.001 ) in the NECT group compared to the IVP group. The above findings are comparable with above studies in respect to stone size.

\section{Treatment}

In the present study, $3(10 \%)$ and $6(20 \%)$ patients respectively in IVU and CT Urography group underwent surgical management whereas 27 $(90 \%)$ and $24(80 \%)$ patients underwent nonsurgical management. This difference was 
statistically not significant. Lee DH et al observed no difference in the urolithiasis treatment plan between the NECT and IVU groups. The incidence of active management, including surgery and extracorporeal shock wave therapy, did not differ between the NECT and IVU groups (35.4 versus $39.9 \%, p=0.67$ ), but the incidence of surgery was higher in the NECT group than in the IVU group (11.8 versus $5.8 \%, p<0.001)$. Among the urolithiasis treatment plans, the proportions of medical expulsion and observed cases did not differ between the NECT and IVU groups. Only $20 \%$ of the patients required surgical management, and $80 \%$ of the patients were treated non-surgically. Thus the findings in our study corroborates with the study done by Lee DH et al.

\section{Specificity \& Sensitivity}

In our study, the sensitivity and specificity of detecting etiology by CTU was $100 \%$ as compared to $66.7 \%$ of IVU. There was significant difference in the accuracy of CTU as compared to IVU. In a study done by Khan $\mathrm{N}$ et al, NCCT comparing with IVU had a higher detection rate for ureterolithiasis, especially for stones in the distal ureter. NCCT compared with IVU also identified more stones in the kidney. NCCT compared with IVU demonstrated a higher detection rate for the number of calculi and related obstruction. The increased number of incidental findings also makes CT more useful. Xie $\mathrm{C}$ et al7found diagnostic accuracy of CTU for UPJO ( Uretero Pelvic Junction Obstruction ) was $85.2 \%$, which was significantly higher than the $49.2 \%$ of IVU. Although suspicious abnormal findings in IVU were recognized in many patients (23/61), they were usually inadequate for making accurate diagnosis and further examinations were thus acquired. Fielding JR et al, Smith RC et al, Ahmad NA et al have reported sensitivity of NECT in evaluating patients with suspected urinary calculi was $97 \%$ to $98 \%$, and its specificity was $96 \%$ to $100 \%$. Song HJ et alreported NECT showed higher sensitivity and specificity than did IVP in this respect, because all urinary tract calculi could be identified by NECT.
In addition, NECT could evaluate the severity of the urinary tract obstruction as well.

We infer from our study and the above studies with similar observations that CTU is more sensitive and specific than IVU in detecting urinary tract calculopathy.

\section{Conclusion}

CTU is better than IVU in detection of urinary stone, save time and is cost effective. Developmental progression on imaging equipment reduced the radiation dose. IVU is replaced by CTU in developed countries and still in use in third world countries. IVU is not ideal modality for diagnosis urinary stone, it is recommended to be completely replaced by CTU with respect to increase the awareness, training and economic development to support and sustain quality health services.

Acknowledgements: None

Source of Support- None

\section{References}

1. Heneghan JP, Kim DH, Leder RA, et al. Compression CT urography: a comparison with IVU in the opacification of the collecting system and ureters.J Comput Assist Tomogr. 2001;25:343-347.

2. Kekelidze M, Dijkshoorn ML, Dwarkasing $\mathrm{S}$, et al. Initial experience and perspectives of a low-dose two-phase triple bolus single scan multidetector CT urography (MDCTU) protocol. Eur Radiol. 2006;16 (Suppl):181.

3. Lim GS, Jang SH, Son JH, Lee JW, Hwang JS, Lim CH, Kim DJ, Cho DS. Comparison of Non-contrast-Enhanced Computed Tomography and Intravenous Pyelogram for Detection of Patients With Urinary Calculi.

4. Bali VM and Lohit HP. Acute ureteric colic: ultrasound as the initial diagnostic tool. American Journal of Oral Medicine and Radiology. 2016;3(1):24-27. 
5. Lee DH, Chang IH, Kim JW, Chi BH, and Park SB. Usefulness of Nonenhanced Computed Tomography for Diagnosing Urolithiasis without Pyuria in the Emergency Department. BioMed Research International. Volume 2015, Article ID 810971, 6 pages

6. Xie C, Guo J, Wang G, Wang $\mathrm{H}$. Comparison between Intravenous Urography and Computed Tomography. Nephro-Urol Mon. 2011;3(4):258-263

7. Erbas G, Oktar S, Kilicc K, Sen I, Budakoglu II, Arac M. Unenhanced urinary CT: value of parenchymal attenuation measurements in differentiating acute vs. chronic renal obstruction. European Journal of Radiology 2012;81(5):825-9.

8. Gupta R, Raghuvanshi S Multi-detector CT Urography in the diagnosis of urinary tract abnormalities. Int $\mathrm{J}$ Med Res Rev 2016;4(2):222-226.

9. Khan N, Anwar Z, Zafar AM, Ahmed F, Ather MH. A comparison of non-contrast CT and intravenous urography in the diagnosis of urolithiasis and obstruction. African Journal of Urology. 2012. 18, 108-111

10. Song HJ, Cho ST, Kim KK. Investigation of the location of the ureteral stone and diameter of the ureter in patients with renal colic. Korean J Urol 2010;51:198-201.

11. Fielding JR, Steele G, Fox LA, Heller H, Loughlin KR. Spiral computerized tomography in the evaluation of acute flank pain: a replacement for excretory urography. J Urol 1997;157:2071-3.

12. CT urography: Silverman's Atlas 1st edition, 2007, chapter three, P23-26.

13. Maher MM, Jhaveri KS, Lucey BC, et al. Does the administration of saline flush during CT urography (CTU) improve ureteric distension and opacification? A prospective study. Radiology. 2001;221 (P):500.

14. Chow LC, Sommer FG. Multidetector CT urography with abdominal compression and three-dimensional reconstruction. AJR Am J Roentgenol. 2001;177:849855.

15. Coakley FV, Yeh BM. Invited commentary. RadioGraphics 2003; 23:1455-1456. [Commentary on: Joffe SA, Servaes S, Okon S, Horowitz M. Multi-detector row CT urography in the evaluation of hematuria. RadioGraphics 2003; 23:1441-1455

16. Dyer RB, Chen MY, Zagoria RJ. Intravenous urography: technique and interpretation. RadioGraphics 2001; 21:799-821.

17. Song HJ, Cho ST, Kim KK. Investigation of the location of the ureteral stone and diameter of the ureter in patients with renal colic. Korean J Urol 2010;51:198-201.

18. Ahmad NA, Ather MH, Rees J. Unenhanced helical computed tomography in the evaluation of acute flank pain. Int $\mathbf{J}$ Urol 2003; 10:287-92.

19. Smith RC, Verga M, McCarthy S, Rosenfield AT. Diagnosis of acute flank pain: value of unenhanced helical CT. AJR Am J Roentgenol 1996;166:97-101. 